\title{
Effects of Degradation of Nonylphenol in Waste Water by Strong Ionization and Its Degradation Products on Sex Differentiation in Zebrafish
}

\author{
Haijun YU, Chengwu YI ${ }^{1}$, Rongjie YI and Liu YANG \\ School of Environment and Safety Engineering, Jiangsu University, Zhenjiang 212013, \\ China
}

\begin{abstract}
Nonylphenol (NP), as a typical environmental endocrine disruptor, exists widely in the natural environment. It has a high toxicity with a low concentration. NP at the level of $\mu \mathrm{g} / \mathrm{L}$ is enough to interfere with the sex differentiation of many aquatic organisms. The effects of degradation of NP in waste water by strong ionization (SID) and its degradation products on sex differentiation in zebrafish was studied in this paper. The NP solution of $5 \mathrm{mg} / \mathrm{L}$ was degraded by SID device, and the $20 \mathrm{~d}$ zebrafish were exposed to NP wastewater of different concentrations before and after degradation until their sexual maturity. The body length, body weight and sex differentiation ratio of zebrafish were recorded, and the sex hormone levels of zebrafish were extracted and detected. The gonadal glands of zebrafish were slices and analysed. This study found that the effects of nonylphenol on male zebrafish were much greater than that of female, and there was no significant positive correlation between toxicity and dose. In addition, the effects of $5 \mathrm{mg} / \mathrm{L} \mathrm{NP}$ wastewater degraded by SID for $60 \mathrm{~min}$ were not significantly different from those of the control group, indicating that SID could effectively degrade NP and alleviate its biological toxicity.
\end{abstract}

Keywords. Strong ionization, waste water, degradation

\section{Introduction}

Nonylphenol is a typical environmental endocrine disruptor, which has been detected in all watersheds in China. In some watersheds, such as the Pearl River (33200ng/L) [1], the concentration is much higher than the $6.6 \mu \mathrm{g} / \mathrm{L}$ standard set by the U.S. Environmental Protection Agency (EPA).NP structure is similar to estradiol, so it can mimic natural estrogen, affect the sex differentiation and reproduction of the organism through interaction with estrogen receptor, thus affecting the dynamic balance of the whole population [2], and damage the liver, kidney and gonad tissues of the organism [3]. Other studies have found that NP can induce cell apoptosis, autophagy and necrosis, and induce the generation of cancer cells to a certain extent [4]. At present, researchers have detected the presence of NP in the decidua and early embryos of

1 Corresponding Author, Chengwu YI, School of Environment and Safety Engineering, Jiangsu University, Zhenjiang 212013, P.R. China; Email: yichengwu0943@163.com. 
pregnant women, and found that NP has obvious characteristics of maternal transfer [5], indicating that NP contamination cannot be ignored.

Due to the high toxicity and stable structure of NP itself, the degradation effect of NP by traditional sewage treatment methods is not ideal. Studies have shown that NP with complex side chain structure has very low biodegradability and can hardly be degraded in SBR [6]. In recent years, some advanced oxidation degradation of refractory organic pollutants in wastewater has become a hot research technology. Traditional advanced oxidation technologies, such as Fenton method, do not have a high degradation efficiency for NP, while some methods combining nanoscale catalysts have improved the degradation efficiency of NP, but the service life [7] and recovery of catalysts [8] have become new problems. Some studies have shown that strong ionization dielectric barrier discharge (SID) has a high removal effect on the refractory organic pollutants in sewage without the addition of reagents or catalysts. Such as pyridine $(20 \mathrm{mg} / \mathrm{L}, 96 \%)$ [9] and bisphenol A $(20 \mathrm{mg} / \mathrm{L}, 99.59 \%)$ [10], the degradation efficiency was higher than $95 \%$. Therefore, in this experiment, the SID method was chosen to degrade NP in sewage, to determine the degradation efficiency of NP by SID method, and to explore the rule of toxicity change before and after NP degradation. In this study, zebrafish were used as the model organism, and zebrafish were exposed to $\mathrm{NP}$ wastewater with different concentrations and degradation time. By measuring the body length and weight of zebrafish, the influence on its growth and development was analysed; By recording the sex ratio and detecting the content of sex hormones to explore the influence of sex differentiation; The influence of gonad development was studied by section observation and pathological analysis of gonad. On the one hand, this experiment is used to verify the previous studies on the pure toxicology of NP, on the other hand, it also improves the shortcomings of the study on pure degradation, and provides a basis for the toxicology study on the degradation of NP by strong ionization discharge.

\section{Experiments}

\subsection{Experimental Device}

In this experiment, SID technology is used to convert the power frequency voltage of $220 \mathrm{~V} / 50 \mathrm{~Hz}$ into the high frequency voltage of $2.5-4 \mathrm{kV} / 15 \sim 23 \mathrm{kHz}$ by high frequency and high voltage power supply and input it to the dielectric barrier discharge (DBD) generator. The raw material $\mathrm{O}_{2}$ is ionized and injected into the water phase through the Venturi nozzle to react with water molecules, forming $\mathrm{O}_{3}$ and $\cdot \mathrm{OH}$ as the main active substances, oxidizing and degrading the pollutants in water, so as to achieve the purpose of purifying organic pollutants.

\subsection{Equipment and Drugs}

UV-visible spectrophotometer, TOC detector, tissue leveller; Ultra low temperature refrigerator; Electronic Balance; Ultra-micro nucleic acid protein analyzer.

NP was purchased from Shanghai AlADDIN Biochemical Technology Co., Ltd, BCA protein concentration determination kit, Fish estradiol (E2) ELISA kit, Fish testosterone (T) ELISA kit from Shanghai New Platinum Chemical Technology Co., 
Ltd. The rest reagents were purchased from Sinopharm Group and were all analytical pure. All solutions were prepared with pure water from the ultra-pure water system (UPPLUS-100L, LICHEN, CHINA).

\subsection{Methods}

\subsubsection{NP Degradation Analysis}

Nonylphenol solution was scanned by UV-Vis spectrophotometer to determine its maximum absorption wavelength, and its concentration was determined by the absorbance at the maximum absorption wavelength. TOC value was measured with TOC detector to determine the degree of mineralization.

\subsubsection{Toxicity Test of Zebrafish}

180 20d AB zebrafish were selected. They were divided into six groups: CK (blank), $\mathrm{CK}+($ solvent control), $20 \mu \mathrm{g} / \mathrm{L}$ (NP concentration was $20 \mu \mathrm{g} / \mathrm{L}), 200 \mu \mathrm{g} / \mathrm{L}$ (NP concentration was $200 \mu \mathrm{g} / \mathrm{L}), 30 \mathrm{~min}(5 \mathrm{mg} / \mathrm{LNP}$ degradation for $30 \mathrm{~min})$, and $60 \mathrm{~min}$ ( $5 \mathrm{mg} / \mathrm{LNP}$ degradation for $60 \mathrm{~min}$ ), with three parallel groups in each group. They were exposed to the corresponding concentration for 60 days. Deformities and death of zebrafish were recorded during exposure period, and body length, weight and sex ratio of each group were measured after exposure. Six zebrafish (three males and three females) were taken from each group, and the contents of estradiol and testosterone were extracted and detected respectively according to the corresponding kit instructions after exposure. The gonadal sections of female and male zebrafish were stained, respectively, and the gonadal sections of female and male zebrafish were photographed under a 100x and 200x microscope for pathological analysis.

\section{Results and Discussion}

\subsection{NP Degradation Analysis}

The SID method was used to degrade the NP wastewater with an initial concentration of $5 \mathrm{mg} / \mathrm{L}$. It was found that the degradation rate of NP reached about $95 \%$ at $30 \mathrm{~min}$, and the characteristic peak of NP was almost not detected at $60 \mathrm{~min}$, so it was completely degraded by default. The results of mineralization analysis by TOC showed that the mineralization rate of NP was about $44.84 \%$ after degradation for $60 \mathrm{~min}$, indicating that many small molecule intermediate products had not been completely degraded into $\mathrm{H}_{2} \mathrm{O}$ and $\mathrm{CO}_{2}$.

\subsection{Toxicity Test of Zebrafish}

\subsubsection{Body Length, Body Weight and Sex Differentiation Ratio of Zebrafish}

There was no obvious deformity and death in each group during exposure. In terms of sex differentiation, we found that $20 \mu \mathrm{g} / \mathrm{L}$ nonylphenol significantly increased the proportion of females in zebrafish $(73 \%)$, while $200 \mu \mathrm{g} / \mathrm{L}$ nonylphenol had no significant effect. It seemed that there was no positive correlation between NP toxicity 
and dose in this respect. Previous studies have also shown that lower concentrations of $\mathrm{NP}$ tend to be more toxic in terms of influencing sex ratio.

The growth and development of zebrafish is one of the important indicators of their reproductive health. Nonylphenol can cause a series of effects on the body length and weight of zebrafish through endocrine disruption. After exposure, the body length and weight of zebrafish in each group were shown in figure 1. Compared with the CK group, the body length and weight of zebrafish in the $20 \mu \mathrm{g} / \mathrm{L}$ group were increased, and the body length and weight of the $200 \mu \mathrm{g} / \mathrm{L}$ and 30min groups were significantly decreased. From the Angle of body length and body weight, it was found that $20 \mu \mathrm{g} / \mathrm{L}$ nonylphenol had a little promotion effect on the growth and development of zebrafish, while $200 \mu \mathrm{g} / \mathrm{L}$ nonylphenol significantly inhibited the growth and development of zebrafish. To some extent, NP promoted the growth and development of zebrafish at low concentration but inhibited it at high concentration.
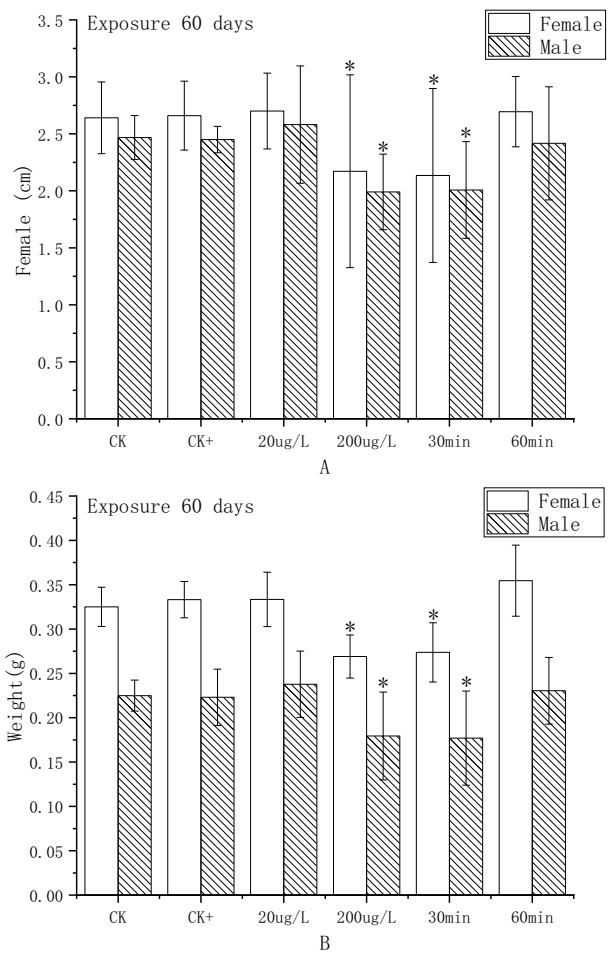

Figure 1. Effects of NP on body length (A) and body weight (B) of zebrafish. Values represent the mean \pm $\mathrm{SD}$ of three replicate samples from each group. ${ }^{*} \mathrm{p}<0.05$ and $* * \mathrm{p}<0.01$ indicate significant differences between exposure groups and $\mathrm{CK}+$ group.

\subsubsection{Sex Hormone Level of Zebrafish}

Estradiol (E2) and testosterone (T) were extracted from zebrafish according to the corresponding kit instructions. The results are shown in figure 2 below. As can be seen from the chart, after exposure, $\mathrm{T}$ in each concentration group in males was significantly lower than that in the control group, especially in the $200 \mu \mathrm{g} / \mathrm{L}$ and degradation group for $30 \mathrm{~min}$, while $\mathrm{T}$ in each concentration group in females was slightly higher than that 
in the control group. Studies have shown that the imbalance of T and E2 ratio will affect gonadal development, sex differentiation and reproduction of fish [11]. In this study, with the increase of NP exposure concentration, E2 increased and T decreased significantly in male zebrafish, and the ratio of T to E2 was unbalanced. Therefore, gonadal development, sex differentiation and reproductive ability would be greatly affected.
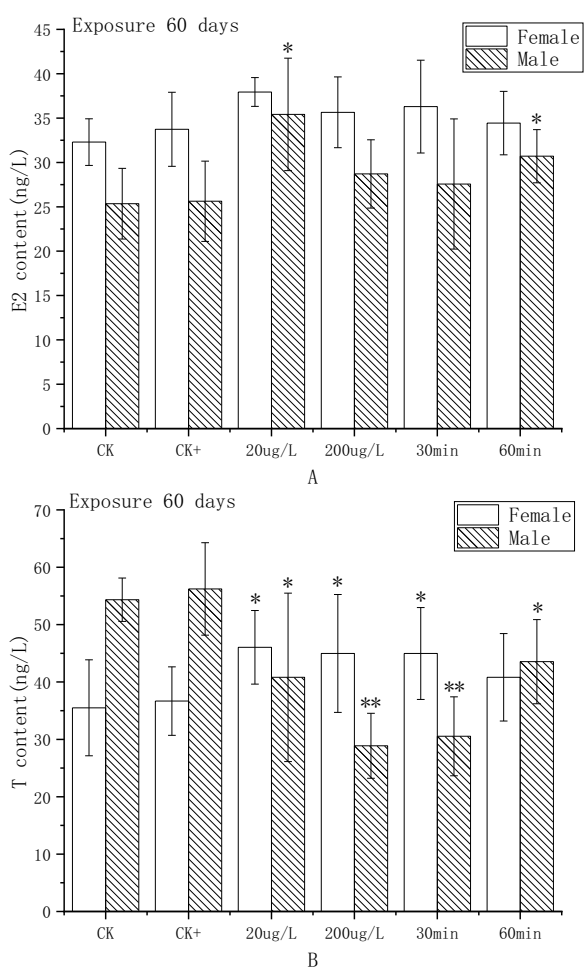

Figure 2. The effect of NP on E2(A) and T(B) of zebrafish. Values represent the mean \pm SD of three replicate samples from each group. ${ }^{*} p<0.05$ and ${ }^{* *} p<0.01$ indicate significant differences between exposure groups and $\mathrm{CK}+$ group.

\subsubsection{Gonadal Development of Zebrafish}

The ovary is the reproductive organ of female animals, where follicle formation, fertilized egg maturation, steroid production, and steroid hormone synthesis occur [12]. In this paper, the ovarian sections of female zebrafish in each group were shown in figure 3 below. In each concentration group, oocyte development at each stage was found, and oocyte development was normal without obvious pathological changes. It can be seen that NP has no significant effect on ovarian development.

In addition, the same analysis was made on the gonadal gland of male zebrafish. As shown in figure 4 of the gonadal section, the testis of zebrafish of $\mathrm{CK}$ and $\mathrm{CK}+$ was covered with a large number of spermatozoa, and each spermatogenic capsule contained germ cells at different stages. Although there were germ cells in different periods in each concentration group, the number of sperm was significantly reduced and intercellular space appeared, especially in the testicular sections of zebrafish in the 
$200 \mu \mathrm{g} / \mathrm{L}$ and $30 \mathrm{~min}$ groups. The number of sperm in spermatogenic vesicles was significantly reduced and fragmented, and a large number of intercellular spaces appeared. Studies have shown that sex hormones can directly regulate sperm production in fish [13], while the imbalance of $\mathrm{T}$ and E2 ratio may interfere with gonadal development, sex differentiation and reproductive function in fish [14]. In this study, the ratio of T to E2 was significantly decreased in both groups of $200 \mu \mathrm{g} / \mathrm{L}$ and $30 \mathrm{~min}$, which may be one of the reasons for the testis lesions of zebrafish.
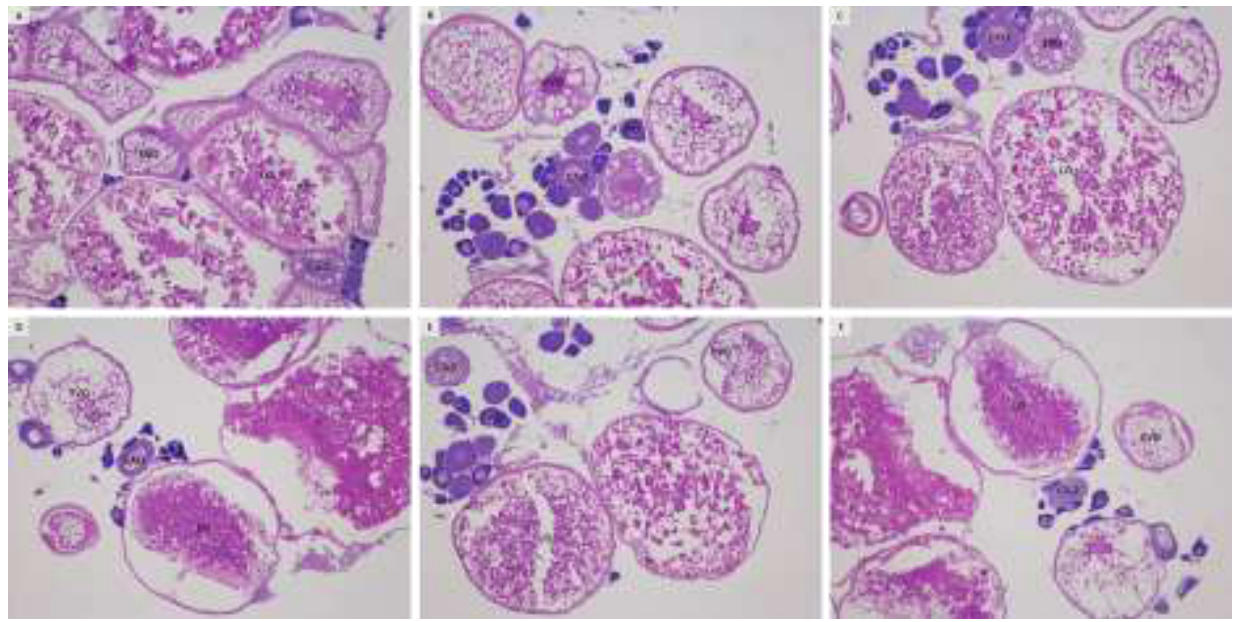

Figure 3. Histological section of zebrafish ovary. (A) Control (B) Control+ (C) $20 \mu \mathrm{g} / \mathrm{L}$ (D) $200 \mu \mathrm{g} / \mathrm{L}$ (E) NP Wastewater degraded by SID for $30 \mathrm{~min}$ (F) NP Wastewater degraded by SID for $60 \mathrm{~min}$. The ovaries were categorized into the stages: perinucleolar oocytes (PO), cortical alveolar oocytes (CAO), early vitellogenic oocytes (EVO), and latemature oocytes $(\mathrm{LMO})(100 \times$ magnification).
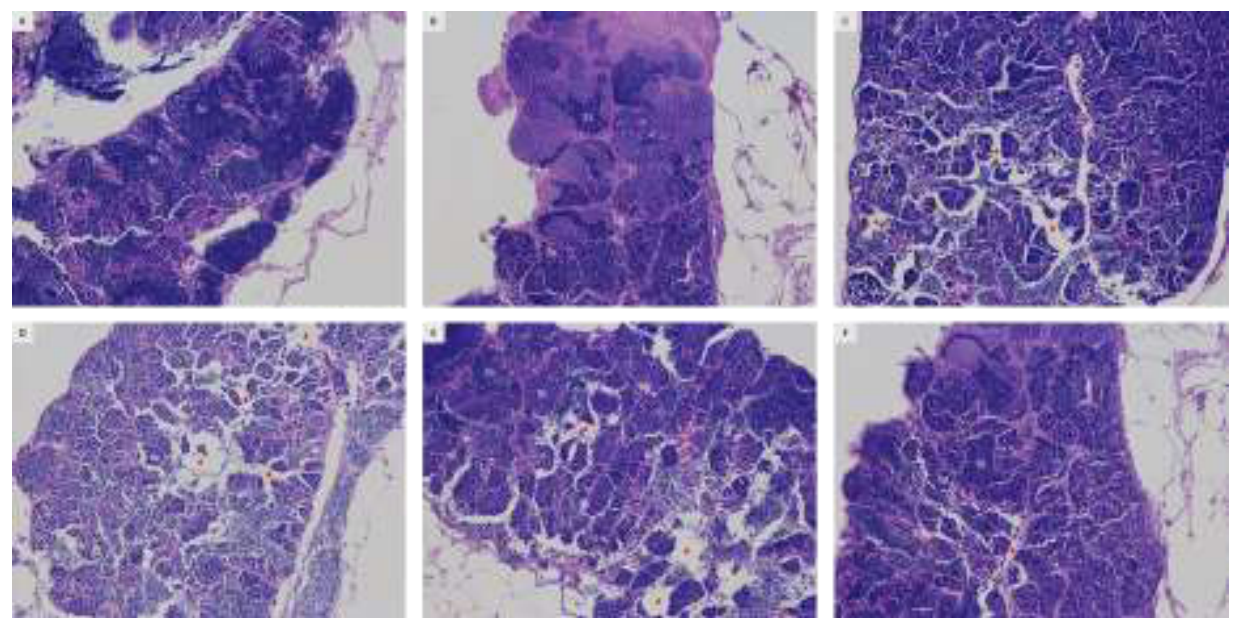

Figure 4. Histological section of zebrafish testis: (A) Control (B) Control+ (C) $20 \mu \mathrm{g} \cdot \mathrm{L}-1$ (D) $200 \mu \mathrm{g} \cdot \mathrm{L}-1$ (E) NP Wastewater degraded by SID for $30 \mathrm{~min}$ (F) NP Wastewater degraded by SID for $60 \mathrm{~min}$. The triangle shows that the cell gap is widened. The testis was categorized into the stages: spermatogonia (Sg), spermatocytes (Sc) and spermatozoa (Sz). (200×magnification) 


\section{Conclusion}

In this paper, the SID method was used to degrade the $5 \mathrm{mg} / \mathrm{L} \mathrm{NP}$ wastewater. After 60 minutes of degradation, the UV-Vis spectrophotometer could not scan the characteristic peak of NP, the mineralization analysis results showed that the mineralization rate was $48.84 \%$, indicating that many small molecular intermediates had not been completely degraded. By exposing zebrafish to NP wastewater with different concentration and degradation time, the toxicological experiments on zebrafish showed that nonylphenol had a great influence on the growth and development of zebrafish, especially male zebrafish: high concentration of nonylphenol significantly inhibited the growth and development of zebrafish. However, the lower concentration of nonylphenol significantly increased the proportion of females in the process of sex differentiation in zebrafish. Male zebrafish had delayed gonad development, reduced sperm count and enlarged testis cavities under the effect of nonylphenol, and these problems became more serious with the increase of NP concentration. The control of each concentration group showed that the effects of 30min group on various indexes of zebrafish were close to those of $200 \mu \mathrm{g} / \mathrm{L}$ group, which was also consistent with the degradation rate of NP. The parameters of the $60 \mathrm{~min}$ degradation group were almost the same as those of the control group, which proved that the small molecular intermediates which had not been completely mineralized in the mineralization analysis had been completely degraded after $24 \mathrm{~h}$ aeration, or had no toxic effects on the growth and development of zebrafish and sex differentiation. It can be inferred that no more toxic intermediates appear in the degradation process of NP by SID technology, and NP in wastewater can be almost completely degraded, which plays a great role in alleviating its environmental toxicity.

\section{References}

[1] Pei G, Li Z, Gibson M and Gao H 2014 Ecological risk assessment of nonylphenol in coastal waters of China based on species sensitivity distribution model Chemosphere 104 113-119.

[2] Sun D, et al 2017 Effect of environmentally-relevant concentrations of nonylphenol on sexual differentiation in zebrafish: A multi-generational study Scientific reports 742907.

[3] Iman S, et al. 2020 Disruptive effects of nonylphenol on reproductive hormones, antioxidant enzymes, and histology of liver, kidney and gonads in Caspian trout smolts Comparative Biochemistry and Physiology Part C 232108756.

[4] Sun C-H, et al. 2019 17a-Ethynylestradiol and 4-nonylphenol stimulate lung adenocarcinoma cell production in xenoestrogenic way Chemosphere 218 793-798.

[5] Chen M, et al. 2016 Occurrence and maternal transfer of chlorinated bisphenol A and nonylphenol in pregnant women and their matching embryos Environmental Science \& Technology 50 (2) 970-977.

[6] Hao R, et al. 2009 Structure-biodegradability relationship of nonylphenol isomers during biological wastewater treatment process Chemosphere 75 987-994.

[7] Bechambi O, Najjar W and Sayadi S 2016 The nonylphenol degradation under UV irradiation in the presence of $\mathrm{Ag}-\mathrm{ZnO}$ nanorods: Effect of parameters and degradation pathway Journal of the Taiwan Institute of Chemical Engineers 60 496-501.

[8] Tang C, et al. 2020 Mechanism investigation on the enhanced photocatalytic oxidation of nonylphenol on hydrophobic $\mathrm{TiO}_{2}$ nanotubes Journal of Hazardous Materials 382121017.

[9] Yang L, et al. 2017 Research on the degradation mechanism of pyridine in drinking water by dielectric barrier discharge Journal of Environmental Sciences (China) 53 238-247.

[10] Geng T, et al. 2020 Mechanism and degradation pathways of bisphenol A in aqueous solution by strong ionization discharge Water, Air, \& Soil Pollution: An International Journal of Environmental Pollution 231 (3) 1596-1606.

[11] Shang E, et al. 2006 Hypoxia affects sex differentiation and development, leading to a male-dominated population in zebrafish (Danio rerio) Environmental Science \& Technology 40 (9) 3118-3140. 
[12] Hannon P R and Flaws J A 2015 The effects of phthala Frontiers in Endocrinology 6 1664-2392.

[13] Liu Z H, et al. 2018 Diethylstilbestrol arrested spermatogenesis and somatic growth in the juveniles of yellow catfish (Pelteobagrus fulvidraco), a fish with sexual dimorphic growth Fish Physiology and Biochemistry 44 (3) 789-803.

[14] Orlando A 2004 Endocrine-disrupting effects of cattle feedlot effluent on an aquatic sentinel species, the fathead minnow Environmental Health Perspectives 112 (5) A270-A270. 